\title{
Commentary \\ Is Placebo Acupuncture What It Is Intended to Be?
}

\author{
Thomas Lundeberg, ${ }^{1}$ Irene Lund, ${ }^{2}$ Audrey Sing, ${ }^{1}$ and Jan Näslund ${ }^{3}$ \\ ${ }^{1}$ Foundation for Acupuncture and Alternative Biological Treatment Methods, Sabbatsbergs Hospital, Sweden \\ ${ }^{2}$ Department of Physiology and Pharmacology, Karolinska Institutet, Sweden \\ ${ }^{3}$ Department of Physiology and Pharmacology, Karolinska Institutet, Stockholm, Sweden \\ Correspondence should be addressed to Jan Näslund, jan.e.naslund@ki.se
}

Received 19 October 2008; Accepted 7 May 2009

Copyright (C) 2011 Thomas Lundeberg et al. This is an open access article distributed under the Creative Commons Attribution License, which permits unrestricted use, distribution, and reproduction in any medium, provided the original work is properly cited.

\begin{abstract}
Randomized, placebo-controlled clinical trials are recommended for evaluation of a treatment's efficacy with the goal of separating the specific effects (verum) from the non-specific ones (placebo). In order to be able to carry out placebo-controlled acupuncture trials, minimal/sham acupuncture procedures and a sham acupuncture needle has been used with the intention of being inert. However, clinical and experimental results suggest that sham/minimal acupuncture is not inert since it is reported that both verum acupuncture and sham/minimal acupuncture induce a significant alleviation of pain. This alleviation is as pronounced as the alleviation obtained with standard treatment and more obvious than the one obtained with placebo medication or by the use of waiting list controls. These results also suggest that sham acupuncture needles evoke a physiological response. In healthy individuals sham acupuncture results in activation of limbic structures, whereas a deactivation is seen in patients with pain, i.e. results from healthy individuals do not reflect what is seen in clinical conditions. Also, depending on the etiology of pain (or any under clinical condition under investigation), the response to sham acupuncture is varying. The acupuncture ritual may also be seen as an emotional focused therapy allowing for psychological re-orientation. Sham needling in such context may be as powerful as verum acupuncture. We recommend that the evaluated effects of acupuncture could be compared with those of standard treatment, also taking the individual response into consideration, before its use or non-use is established.
\end{abstract}

\section{Introduction}

During the last decade, a large number of randomized controlled trials (RCT) have been published comparing manual acupuncture or electro-acupuncture with different modes of intended placebo controlled procedures in the treatment of perceived pain. The placebo control procedures most commonly used include minimal or superficial acupuncture (needling of the skin), sham acupuncture (deep or superficial needling of non-acupuncture points) and the use of placebo acupuncture needles (a blunt tip of a needle touches the skin without penetrating it) [1-3]. The intention of these RCTs is to reduce the presence of bias of the results by comparing the size of the interventional specific effects, by means of assumed, specific mechanisms, with the nonspecific effects of an inert (placebo) comparator applied in a placebo-controlled procedure. This trial design is considered the gold standard in evaluation of all types of intervention and its result forms the basis for further determination of the effectiveness of acupuncture.
Recent studies performed in Germany have evaluated the effects of minimal acupuncture (superficial needling outside disease specific acupuncture points according to traditional Chinese medicine-TCM) and acupuncture (needles inserted into classical acupuncture points according to TCM, and manually stimulated until the dull radiation sensation of deqi was evoked) in patients with migraine, low back pain and knee osteoarthritis pain. In general, there was no marked difference in efficacy between minimal acupuncture and acupuncture [4]. Thus, most of the effects of acupuncture have by some been attributed to unspecific placebo responses [5]. However, another possibility is that minimal acupuncture produces specific effect and is consequently not inert. If so, the present research trial design (placebo acupuncture versus acupuncture) is not valid. Furthermore, instead of reducing bias there is a risk of introducing bias against the findings of the tested treatment. This suggestion is supported by the findings of the German RCTs, Tables 1, 2, and 3, showing that placebo acupuncture (here specified as minimal acupuncture) is as effective as standard treatment and more 
TABLE 1: Results, treatment of migraine.

\begin{tabular}{ll}
\hline $\begin{array}{l}\text { Intervention: Author, year (ref.); } \\
\text { number of included subjects }(n)\end{array}$ & $\begin{array}{l}\text { Proportion patients (in } \\
\text { \%) reporting reduced } \\
\text { frequency days with } \\
\text { migraine after treatment } \\
\text { [5-9] }\end{array}$ \\
\hline Acupuncture & 51 \\
$\quad$ Linde, 2005 [6]; $n=302$ & 61 \\
Streng, 2006 [7]; $n=114$ & 47 \\
Diener, 2006 [8];n=960 & \\
Minimal acupuncture & 53 \\
Linde, 2005 [6]; $n=302$ & 39 \\
Diener, 2006 [8]; $n=960$ & \\
Medication & 46 (Flunarizin 5 mg) \\
Diener, 2002 [9]; $n=808$ & 53 (Flunarizin 10 mg) \\
& 48 (Propanolol) \\
$\quad$ van der Key, 2002 [10]; $n=2013$ & 46 (Propanolol) \\
Streng, 2006 [7]; $n=114$ & 49 (Metropolol) \\
Diener, 2006 [8]; $n=960$ & 40 (Standard medication) \\
Placebo tablet & \\
van der Key, 2002 [10]; $n=2013$ & 24 \\
Waiting list & \\
Linde, 2005 [6]; $n=302$ & 15 \\
\hline
\end{tabular}

effective than placebo medication in reducing migraine [6-10]. Interestingly, in low back pain [11-13] and knee osteoarthritis pain [14-16], acupuncture had a better effect as compared to placebo acupuncture. The divergent results in the different pain conditions could be interpreted as that the effects of the sensory stimulation produced by placebo acupuncture and acupuncture is dependent on the etiology of pain. Also, the minimal acupuncture technique is only minimal in a TCM perspective. In a psycho-physiological perspective minimal acupuncture is anything but inert.

\section{Etiology of Pain}

Pain is often considered to be a homogeneous sensory entity, mediated by a specialized high threshold sensory system, which extends from the periphery through the spinal cord, brain stem and thalamus to the cerebral cortex. However, multiple mechanisms have been detected in the nervous system responsible for pain of different etiologies [17]. When pain is transformed from an acute (an alarm signal) to a chronic state (a sustained challenge) part of the brain areas is re-organized. Furthermore, this reorganization continuous also in the chronic state, having an impact on the cortex producing unique pattern [18]. Apart from the pain influence on the cortical function there is a continuous reorganization affecting the supraspinal brain areas responsible for descending modulation of pain. Acute and chronic pain also has different impact on learning and memory [18], which should be considered when assessing the efficacy of acupuncture.
TABLE 2: Results, treatment of low back pain.

\begin{tabular}{ll}
\hline & $\begin{array}{l}\text { Proportion patients } \\
\text { (in\%) reporting } \\
\text { decreased low back pain }\end{array}$ \\
$\begin{array}{l}\text { Intervention: Author, year (ref.); } \\
\text { number of included subjects }(n)\end{array}$ & $\begin{array}{l}{[10-12] \text { and increased }} \\
\text { function [11, 12] after } \\
\text { treatment }\end{array}$ \\
\hline Acupuncture & 54 \\
$\quad$ Brinkhaus, 2006 [11]; $n=298$ & 48 \\
$\quad$ Haake, 2007 [12]; $n=1162$ & 53 \\
$\quad$ Witt, 2006 [13]; $n=3093$ & \\
Minimal acupuncture & 39 \\
$\quad$ Brinkhaus, 2006 [11]; $n=298$ & 44 \\
$\quad$ Haake, 2007 [12]; $n=1162$ & \\
Standard treatment & 27 \\
$\quad$ Haake, 2007 [12]; $n=1162$ & \\
Routine care & \\
$\quad$ Witt, 2006 [13]; $n=3093$ & \\
Waiting list &
\end{tabular}

TABLE 3: Results, treatment of knee osteoarthritis pain.

\begin{tabular}{ll}
\hline $\begin{array}{l}\text { Intervention: Author, year (ref.); } \\
\text { number of included subjects }(n)\end{array}$ & $\begin{array}{l}\text { Proportion patients (in\%) } \\
\text { reporting decreased knee } \\
\text { osteoarthritis pain and } \\
\text { increased function } \\
{[13-15]}\end{array}$ \\
\hline Acupuncture & 52 \\
$\quad$ Witt, 2005 [14]; $n=294$ & 53 \\
$\quad$ Scharf, 2006 [15]; $n=1007$ & 35 \\
$\quad$ Witt, 2006 [16]; $n=712$ & \\
Minimal acupuncture & 28 \\
$\quad$ Witt, 2005 [14]; $n=294$ & 51 \\
$\quad$ Scharf, 2006 [15]; $n=1007$ & \\
Standard care & 29 \\
$\quad$ Scharf, 2006 [15]; $n=1007$ & \\
Routine care & \\
$\quad$ Witt, 2006 [16]; $n=712$ & \\
Waiting list & \\
$\quad$ Witt, 2005 [14]; $n=294$ &
\end{tabular}

\section{Placebo}

A positive placebo response is seen in a varying degree in patients with pain, Parkinson's disease and depression. The placebo response has been reported being more pronounced with invasive procedures or advanced disease [19-21] and, neuroimaging studies have provided a major contribution to our understanding of the mechanisms of the placebo effects. Expectation of symptom improvement has long been believed to play a critical role in the placebo effect and is suggested to be driven by frontal cortical areas, particularly the dorsolateral prefrontal, orbitofrontal and anterior cingulate 
cortices. The ventral striatum is involved in the expectation of rewarding stimuli and, together with the prefrontal cortex, has also been shown to play an important role in the placeboinduced expectation of therapeutic benefit. Also, positron emission tomography studies have shown that the placebo effect is related to the activation of the limbic circuitry. The observation that placebo administration induces the release of dopamine in the ventral striatum of patients with Parkinson's disease suggests a link between the placebo effect and reward mechanisms. In addition to Parkinson's disease, the placebo-reward model may also apply to other disorders. However, the relative contribution of the different neurotransmitters and neuropeptides that are known to be involved in modulating the activity of the limbic system may be disease-specific. Thus, while the placebo-induced clinical benefit observed in Parkinson's disease would mostly reflect the release of dopamine in the dorsal striatum, the activation of opioid and serotonin pathways could be particularly implicated in mediating placebo responses encountered in pain and depression, respectively [19-21].

\section{The Physiological Complexity of Acupuncture Effects}

Acupuncture is a non-uniform treatment where for instance all, Modern, physiological and Traditional, approaches is used. Besides, depending on how it is carried out, different results may be obtained [22]. Responses to the induced stimulation in different endogenous systems have been shown to play key roles in acupuncture analgesia such as the endogenous opioid system and the descending serotoninergic inhibitory pathway [23]. However, as described above, the basic function of these systems have been shown to be altered as a consequence of the pain that also is depending on the etiology of the pain, explaining why different modes of acupuncture, including low and high frequency electroacupuncture, may have different effects (i.e., being the most effective modality). Except endogenous opioids and serotonin, the cholecystokinin octapeptide (CCK-8) has been shown to be important in the effects of acupuncture including development of tolerance. The individual differences of acupuncture analgesia are also associated with inherited genetic factors and the density of CCK receptors. Furthermore, depending on the characteristics of the pain, such as spontaneous, persistent or stimulus-evoked, and its related default mode of the brain, different modalities of acupuncture including the stimuli evoked by placebo acupuncture may have different effects in the patients [24]. In general, patients with musculoskeletal pain of inflammatory or ischemic origin showed the largest probability to obtain pain alleviation whereas in patients with neuropathic pain this was less likely and even so less in patients with persistent (idiopathic) pain [24].

\section{Effects of Placebo Acupuncture in Pain and Other Conditions}

Several studies have reported that the placebo acupuncture procedure is adequate from an expectation perspective, that is, acupuncture naïve patients cannot reveal which is real and which is control. During placebo acupuncture procedures, stimulation of the cutaneous touch receptors and/or skin nociceptors may be activated $[25,26]$. The activity set up in these receptors is conveyed into the brain and result in the modulation of the activity in the brain areas included in the pain neuromatrix such as the limbic structures [2730]. In healthy subjects, the acupuncture results in increased activity in the limbic structures whereas in patients with pain a deactivation of the same structures is reported [3133]. This would suggest that trials using healthy subjects is of great interest but have limited clinical relevance. Also, pains with different etiology may be associated with different characteristics, like spontaneous or stimulus evoked pain. These symptoms may be differently susceptible to the influence of acupuncture. In pain patients with persistent pain the stimulus response to most modalities of sensory stimulations is augmented (central sensitization), whereby for example light stimulation of the skin is perceived as being very strong and in some cases even painful [34]. Also, in patients with persistent pain the receptive fields of central nociceptive neurons are expanded, resulting in a larger topographic distribution in the "higher" levels of central nervous system of the pain [21].

Except for modulating the activity in the hypothalamus and the limbic structures, the activity set up in afferent nerves during placebo acupuncture modulates the reward system resulting in a sensation of wellbeing [35-39]. It can also be assumed that the clinical context of the acupuncture treatment may serve as behavioral conditioning suggesting that the (repeated) needling ritual per se contributes to the therapeutic effects of acupuncture [40]. In a psychological perspective acupuncture may be viewed as an emotion focused therapy were placebo acupuncture or acupuncture results in alterations in the functional connectivity making the patient more susceptible to an emotional re-orientation [41]. One factor that has been suggested to explain a major part of the effects of sham acupuncture in irritable bowel syndrome (IBS) is the interaction between the therapist and the patient [42]. However, this interaction is in itself also context dependent making general conclusions about sham acupunctures efficacy in patient-therapist relations in other conditions invalid [43] since patients treated with acupuncture due to allergic rhinitis physician characteristics played a minor role in the effectiveness of acupuncture treatment [44].

In IBS patients treated with acupuncture or placebo acupuncture the quality of life improved in both groups with no group differences. Furthermore, a more pronounced salivary cortisol decreased was seen in the acupuncture group as was the decrease of the heart rate response during orthostatic stress indicating an increased parasympathetic tone in the acupuncture group. Also, improvement of pain was positively associated with increased parasympathetic tone in the acupuncture group but not in the placebo group. Thus, different mechanisms seem to be involved in placebo acupuncture and acupuncture driven improvements [45]. That cortisol release is decreased after acupuncture is further supported by a study on patients with chronic low back 
pain, subjected to different modalities of acupuncture (traditional Chinese acupuncture, sham acupuncture, electroacupuncture and electro-acupuncture at non-acupuncture points) [46]. A significant decrease in plasma cortisol concentration was measured after all interventions suggesting that the decrease was a centrally controlled, hypothalamic medicated response. However, the changes in cortisol concentrations in plasma are dependent on the condition treated. In patients suffering from "environmental illness" and treated with acupuncture or placebo acupuncture, cortisol concentrations increased. In that study both groups improved significantly during and after treatment without any group differences. The changes seen in biological variables were gradual with a continuous increase in serum cortisol and a decrease in neuropeptide Y [47].

Recently, the use of acupuncture during in vitro fertilization (IVF) treatments has attracted interest as it has been suggested to improve outcome, that is, pregnancy rate. In a randomized double blind study, aimed to compare acupuncture with placebo acupuncture in patients undergoing IVF treatment, 370 patients were randomly allocated to either acupuncture or placebo acupuncture before embryo transfer. Interestingly the overall pregnancy rate was significantly higher in the placebo acupuncture group than that in the acupuncture group resulting in the suggestion that "Placebo acupuncture may not be inert" [48].

\section{Conclusion}

Acupuncture as used currently is not a standardized treatment and likely several acupuncture techniques owns the potential of inducing clinical treatment effects depending on the condition treated. Experimental and clinical studies have shown that the acupuncture placebo procedures applied are not inert, (from a psycho-physiological perspective) and should therefore not be interpreted as placebo-controls in RCTs for the test of efficacy, that is, the present research trial design (placebo acupuncture versus acupuncture) may be questioned. Instead of reducing bias, it introduces a bias against the findings of the acupuncture treatment. The introduction of the placebo needle was a brilliant idea, however, it is up to the user to determine what its use may reflect and how its effect should be interpreted in an evidenced based medicine perspective $[49,50]$. The minimal acupuncture technique may be regarded a valid control of TCM medicated effects but not for effects of acupuncture as a modality of sensory stimulation.

\section{References}

[1] P. White, B. Golianu, C. Zaslawski, and C. Seung-Hoon, "Standardization of nomenclature in acupuncture research (SoNAR)," Evidence-Based Complementary and Alternative Medicine, vol. 4, no. 2, pp. 267-270, 2007.

[2] F. Dincer and K. Linde, "Sham interventions in randomized clinical trials of acupuncture-a review," Complementary Therapies in Medicine, vol. 11, no. 4, pp. 235-242, 2003.

[3] S. Birch, "A review and analysis of placebo treatments, placebo effects, and placebo controls in trials of medical procedures when sham is not inert," Journal of Alternative and Complementary Medicine, vol. 12, no. 3, pp. 303-310, 2006.

[4] "Richtlinie Methoden vertragsarztliche Versorgung (Akupunktur/siehe Beschluss vom 19.09.2006)," 2006, http://www.g-ba .de/informationen/beschluesse/295/.

[5] S. Singh and E. Ernst, Trick or Treatment: The Undeniable Facts about Alternative Medicine, WW Norton, New York, NY, USA, 2008.

[6] K. Linde, A. Streng, S. Jürgens et al., "Acupuncture for patients with migraine: a randomized controlled trial," Journal of the American Medical Association, vol. 293, no. 17, pp. 2118-2125, 2005.

[7] A. Streng, K. Linde, A. Hoppe et al., "Effectiveness and tolerability of acupuncture compared with metoprolol in migraine prophylaxis," Headache, vol. 46, no. 10, pp. 14921502, 2006.

[8] H. C. Diener, K. Kronfeld, G. Boewing et al., "Efficacy of acupuncture for the prophylaxis of migraine: a multicentre randomised controlled clinical trial," The Lancet Neurology, vol. 5, pp. 310-316, 2006.

[9] H. C. Diener, J. Matias-Guiu, E. Hartung et al., "Efficacy and tolerability in migraine prophylaxis of flunarizine in reduced doses: a comparison with propranolol $160 \mathrm{mg}$ daily," Cephalalgia, vol. 22, no. 3, pp. 209-221, 2002.

[10] P.-H. M. Van Der Kuy and J. J. H. M. Lohman, "A quantification of the placebo response in migraine prophylaxis," Cephalalgia, vol. 22, no. 4, pp. 265-270, 2002.

[11] B. Brinkhaus, C. M. Witt, S. Jena et al., "Acupuncture in patients with chronic low back pain: a randomized controlled trial," Archives of Internal Medicine, vol. 166, no. 4, pp. 450457, 2006.

[12] M. Haake, H.-H. Müller, C. Schade-Brittinger et al., "German Acupuncture Trials (GERAC) for chronic low back pain: randomized, multicenter, blinded, parallel-group trial with 3 groups," Archives of Internal Medicine, vol. 167, no. 17, pp. 1892-1898, 2007.

[13] C. M. Witt, S. Jena, D. Selim et al., "Pragmatic randomized trial evaluating the clinical and economic effectiveness of acupuncture for chronic low back pain," American Journal of Epidemiology, vol. 164, no. 5, pp. 487-496, 2006.

[14] C. Witt, B. Brinkhaus, S. Jena et al., "Acupuncture in patients with osteoarthritis of the knee: a randomised trial," Lancet, vol. 366, no. 9480, pp. 136-143, 2005.

[15] H.-P. Scharf, U. Mansmann, K. Streitberger et al., "Acupuncture and knee osteoarthritis: a three-armed randomized trial," Annals of Internal Medicine, vol. 145, no. 1, pp. 12-20, 2006.

[16] C. M. Witt, S. Jena, B. Brinkhaus, B. Liecker, K. Wegscheider, and S. N. Willich, "Acupuncture in patients with osteoarthritis of the knee or hip: a randomized, controlled trial with an additional nonrandomized arm," Arthritis and Rheumatism, vol. 54, no. 11, pp. 3485-3493, 2006.

[17] T. Lundeberg and I. Lund, "Is there a role for acupuncture in endometriosis pain, or 'endometrialgia'?" Acupuncture in Medicine, vol. 26, no. 2, pp. 94-110, 2008.

[18] A. V. Apkarian, M. N. Baliki, and P. Y. Geha, "Towards a theory of chronic pain," Progress in Neurobiology, vol. 87, no. 2, pp. 81-97, 2009.

[19] N. J. Diederich and C. G. Goetz, "The placebo treatments in neurosciences: new insights from clinical and neuroimaging studies," Neurology, vol. 71, no. 9, pp. 677-684, 2008.

[20] S. C. Lidstone and A. J. Stoessl, "Understanding the placebo effect: contributions from neuroimaging," Molecular Imaging and Biology, vol. 9, no. 4, pp. 176-185, 2007. 
[21] P. Enck, F. Benedetti, and M. Schedlowski, "New insights into the placebo and nocebo responses," Neuron, vol. 59, no. 2, pp. 195-206, 2008.

[22] T. Lundeberg, T. Hurtig, S. Lundeberg, and M. Thomas, "Long-term results of acupuncture in chronic head and neck pain," Pain Clinic, vol. 2, no. 1, pp. 15-31, 1988.

[23] Z.-Q. Zhao, "Neural mechanism underlying acupuncture analgesia," Progress in Neurobiology, vol. 85, no. 4, pp. 355-375, 2008.

[24] M. Thomas and T. Lundeberg, "Does acupuncture work?" Pain Clinical Updates, vol. 4, pp. 1-11, 1996.

[25] T. Lundeberg, I. Lund, J. Näslund, and M. Thomas, "The emperor's sham-wrong assumption that sham needling is sham," Acupuncture in Medicine, vol. 26, no. 4, pp. 239-242, 2008.

[26] I. Lund, J. Näslund, and T. Lundeberg, "Minimal acupuncture is not a valid placebo control in randomised controlled trials of acupuncture: a physiologist's perspective," Chinese Medicine, vol. 4, article no. 1, 2009.

[27] R. P. Dhond, N. Kettner, and V. Napadow, "Do the neural correlates of acupuncture and placebo effects differ?" Pain, vol. 128, no. 1-2, pp. 8-12, 2007.

[28] H. MacPherson, G. Green, A. Nevado et al., "Brain imaging of acupuncture: comparing superficial with deep needling," Neuroscience Letters, vol. 434, no. 1, pp. 144-149, 2008.

[29] H. Olausson, Y. Lamarre, H. Backlund et al., "Unmyelinated tactile afferents signal touch and project to insular cortex," Nature Neuroscience, vol. 5, no. 9, pp. 900-904, 2002.

[30] J. Cole, M. C. Bushnell, F. McGlone et al., "Unmyelinated tactile afferents underpin detection of low-force monofilaments," Muscle and Nerve, vol. 34, no. 1, pp. 105-107, 2006.

[31] V. Napadow, N. Kettner, J. Liu et al., "Hypothalamus and amygdala response to acupuncture stimuli in carpal tunnel syndrome," Pain, vol. 130, no. 3, pp. 254-266, 2007.

[32] A. Poznanski, M. Hsu, R. Gracely, C. Daniel, and R. Harris, "Differences in central neural pain processing following acupuncture and sham acupuncture therapy in fibromyalgia (FM)," in Proceedings of the American Pain Society's 27th Annual Scientific Meeting, 2008, Poster \#298, Abstract 8290.

[33] J. Fang, Z. Jin, Y. Wang et al., "The salient characteristics of the central effects of acupuncture needling: limbic-paralimbicneocortical network modulation," Human Brain Mapping, vol. 30, no. 4, pp. 1196-1206, 2009.

[34] T. Lundeberg and I. Lund, "Are reviews based on sham acupuncture procedures in fibromyalgia syndrome (FMS) valid?" Acupuncture in Medicine, vol. 25, no. 3, pp. 100-106, 2007.

[35] V. Faria, M. Fredrikson, and T. Furmark, "Imaging the placebo response: a neurofunctional review," European Neuropsychopharmacology, vol. 18, no. 7, pp. 473-485, 2008.

[36] D. J. Scott, C. S. Stohler, C. M. Egnatuk, H. Wang, R. A. Koeppe, and J.-K. Zubieta, "Individual differences in reward responding explain placebo-induced expectations and effects," Neuron, vol. 55, no. 2, pp. 325-336, 2007.

[37] J. Pariente, P. White, R. S. J. Frackowiak, and G. Lewith, "Expectancy and belief modulate the neuronal substrates of pain treated by acupuncture," NeuroImage, vol. 25, no. 4, pp. 1161-1167, 2005.

[38] R. Barker Bausell, L. Lao, S. Bergman, W.-L. Lee, and B. M. Berman, "Is acupuncture analgesia an expectancy effect? Preliminary evidence based on participants' perceived assignments in two placebo-controlled trials," Evaluation and the Health Professions, vol. 28, no. 1, pp. 9-26, 2005.
[39] T. Lundeberg, I. Lund, and J. Näslund, "Acupuncture-selfappraisal and the reward system," Acupuncture in Medicine, vol. 25, no. 3, pp. 87-99, 2007.

[40] T. Lundeberg and I. Lund, "Acupuncture for preconditioning of expectancy and/or Pavlovian extinction," Acupuncture in Medicine, vol. 26, no. 4, pp. 234-238, 2008.

[41] J. T. Ehrenreich, C. P. Fairholme, B. A. Buzzella, K. K. Ellard, and D. H. Barlow, "The role of emotion in psychological therapy," Clinical Psychology, vol. 14, no. 4, pp. 422-428, 2007.

[42] T. J. Kaptchuk, J. M. Kelley, L. A. Conboy et al., "Components of placebo effect: randomised controlled trial in patients with irritable bowel syndrome," British Medical Journal, vol. 336, no. 7651, pp. 999-1003, 2008.

[43] C. Paterson and N. Britten, "The patient's experience of holistic care: insights from acupuncture research," Chronic lllness, vol. 4, no. 4, pp. 264-277, 2008.

[44] B. Brinkhaus, C. M. Witt, S. Jena, B. Liecker, K. Wegscheider, and S. N. Willich, "Acupuncture in patients with allergic rhinitis: a pragmatic randomized trial," Annals of Allergy, Asthma and Immunology, vol. 101, no. 5, pp. 535-543, 2008.

[45] A. Schneider, C. Weiland, P. Enck et al., "Neuroendocrinological effects of acupuncture treatment in patients with irritable bowel syndrome," Complementary Therapies in Medicine, vol. 15, no. 4, pp. 255-263, 2007.

[46] H. Harbach, B. Moll, R.-H. Boedeker et al., "Minimal immunoreactive plasma $\beta$-endorphin and decrease of cortisol at standard analgesia or different acupuncture techniques," European Journal of Anaesthesiology, vol. 24, no. 4, pp. 370376, 2007.

[47] B. B. Arnetz, M. Berg, I. Anderzen, T. Lundeberg, and E. Haker, "A nonconventional approach to the treatment of 'environmental illness," Journal of Occupational and Environmental Medicine, vol. 37, no. 7, pp. 838-844, 1995.

[48] E. W. S. So, E. H. Y. Ng, Y. Y. Wong, E. Y. L. Lau, W. S. B. Yeung, and P. C. Ho, "A randomized double blind comparison of real and placebo acupuncture in IVF treatment," Human Reproduction, vol. 24, no. 2, pp. 341-348, 2009.

[49] K. Streitberger and J. Kleinhenz, "Introducing a placebo needle into acupuncture research," Lancet, vol. 352, no. 9125, pp. 364-365, 1998.

[50] H. Yamashita and H. Tsukayama, "Minimal acupuncture may not always minimize specific effects of needling," The Clinical Journal of Pain, vol. 17, p. 277, 2001. 


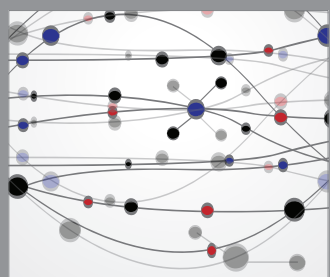

The Scientific World Journal
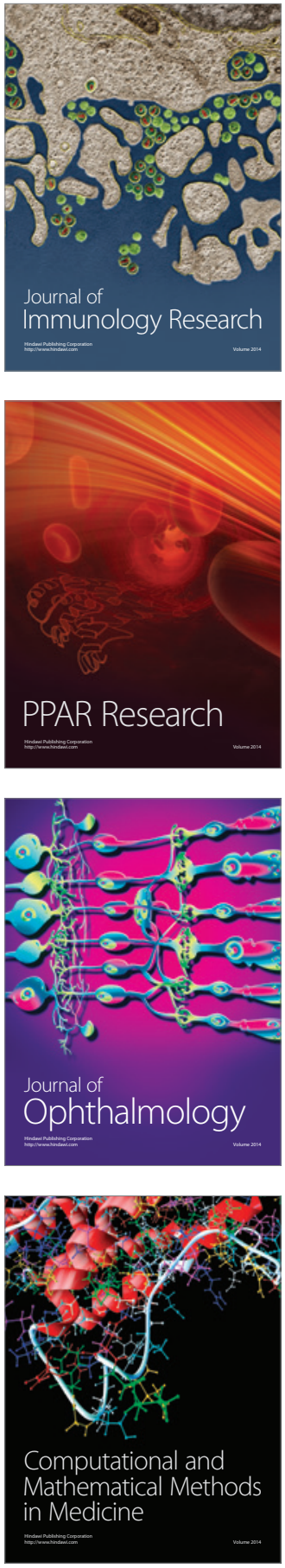

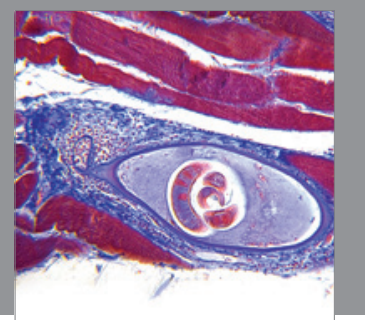

Gastroenterology

Research and Practice
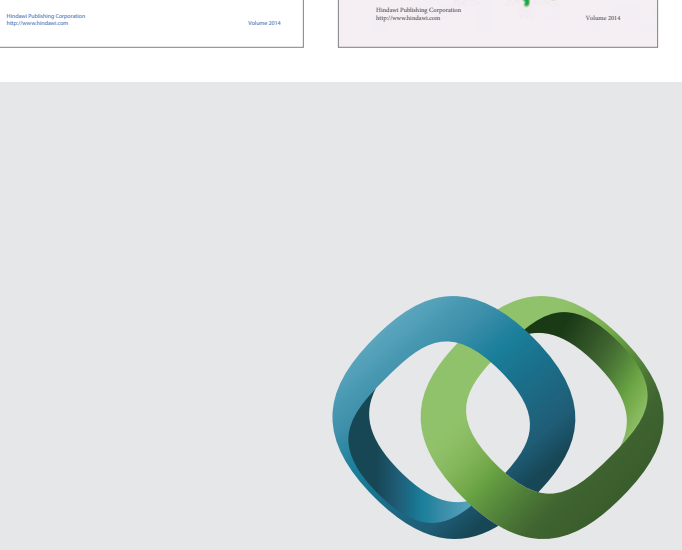

\section{Hindawi}

Submit your manuscripts at

http://www.hindawi.com
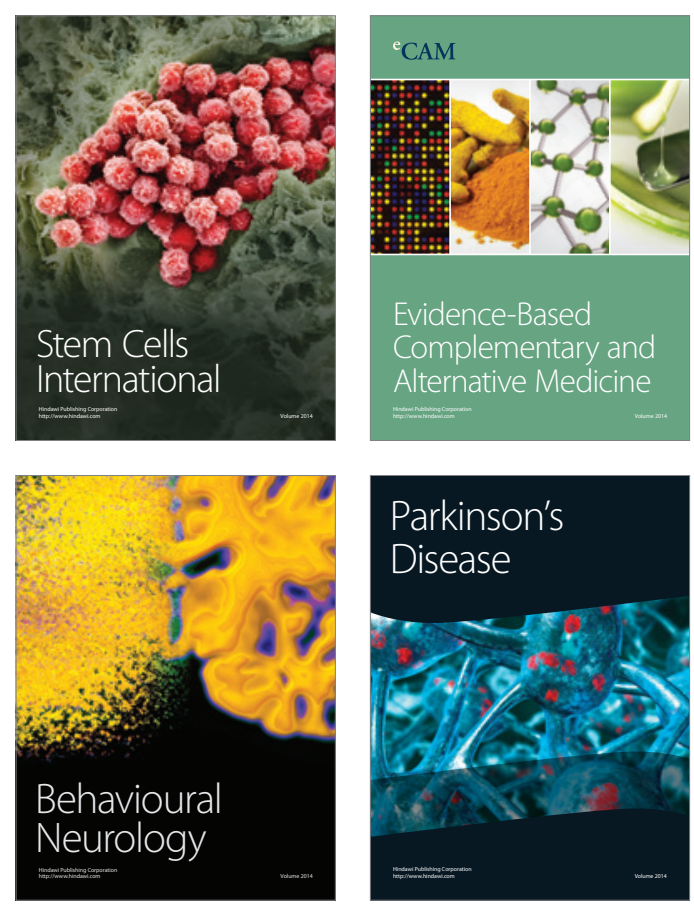

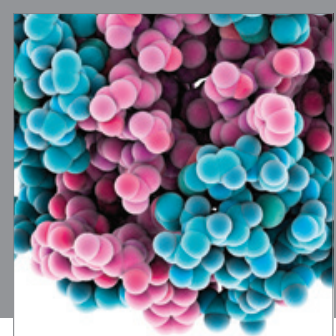

Journal of
Diabetes Research

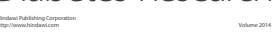

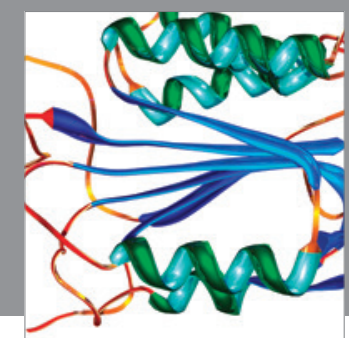

Disease Markers
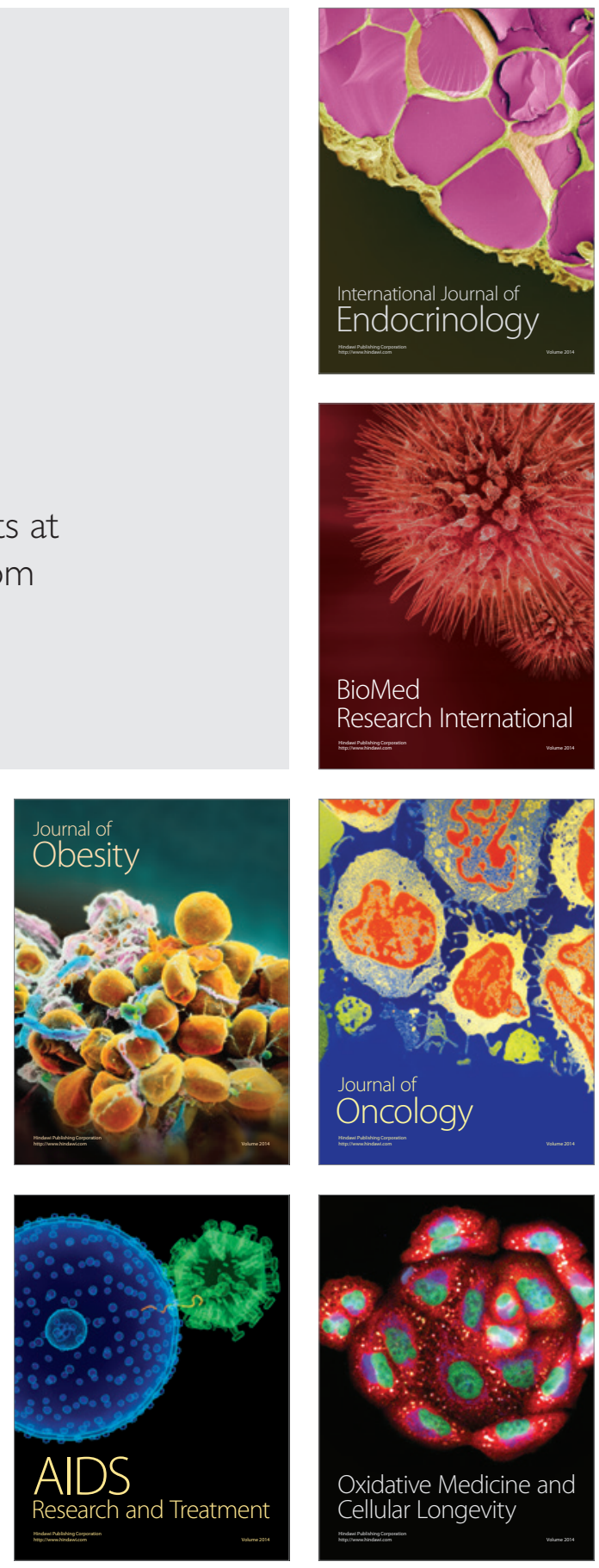